\title{
NOTE ON THE USE OF FRACTIONAL INTEGRATION OF BESSEL FUNCTIONS*
}

\author{
BY H. P. THIELMAN
}

1. Introduction. In a recent paper, W. O. Pennell $\dagger$ pointed out that under certain conditions fractional integration and differentiation of trigonometric functions will lead to Bessel functions and vice versa. Making use of this fact he was able to convert known expansions in sines and cosines into expansions in Bessel functions, and known expansions in Bessel functions into trigonometric expansions by the simple process of termby-term differentiation and integration. In this note it is shown that in some cases fractional integration of Bessel functions leads to the squares of such functions. Use is then made of this fact in order to derive a number of expansions in squares of Bessel functions by fractionally integrating known expansions in Bessel functions.

2. Fractional Integration of Bessel Functions. Fractional integration $\ddagger$ with the Heaviside operator $p$ is defined by the equation

$$
p^{-v} f(x)=\int_{0}^{x} \frac{(x-t)^{\nu-1}}{\Gamma(\nu)} f(t) d t
$$

where $\nu>0$. Fractional differentiation is given by

$$
p^{v} f(x)=\frac{d^{b}}{d x^{b}} \int_{0}^{x} \frac{(x-t)^{c-1}}{\Gamma(c)} f(t) d t
$$

where $\nu>0,0<c<1, b$ is a positive integer, and $\nu=b-c$.

If in the well known Neumann's integral§

* Presented to the Society, April 7, 1934.

$\dagger \mathrm{W}$. O. Pennell, The use of fractional integration and differentiation for obtaining certain expansions in terms of Bessel functions or of sines and cosines, this Bulletin, vol. 38 (1932), pp. 115-122.

$\ddagger$ For a bibliography on fractional integration and differentiation see $H$. T. Davis, The application of fractional operators to functional equations, American Journal of Mathematics, vol. 49 (1927), pp. 123-142.

$\S$ See G. N. Watson, Theory of Bessel Functions, p. 150. 


$$
J_{\mu}(z) J_{\nu}(z)=\frac{2}{\pi} \int_{0}^{\pi / 2} J_{\mu+\nu}(2 z \cos \theta) \cos (\mu-\nu) \theta d \theta,
$$

where $\mu+\nu>-1$, we set $\mu=\nu, z=a x^{1 / 2}, \cos \theta=t^{1 / 2} x^{1 / 2}$, we obtain

$$
J_{\nu}^{2}\left(a x^{1 / 2}\right)=\frac{1}{\pi} \int_{0}^{x} J_{2 \nu}\left(2 a t^{1 / 2}\right)(x-t)^{-1 / 2} t^{-1 / 2} d t, \quad(\nu>-1 / 2),
$$

which by (1) is seen to be equivalent to

$$
p^{-1 / 2} x^{-1 / 2} J_{2 \nu}\left(2 a x^{1 / 2}\right)=\pi^{1 / 2} J_{\nu}^{2}\left(a x^{1 / 2}\right), \quad(\nu>-1 / 2) .
$$

Here $J_{\nu}(z)$ is the Bessel function of the first kind of order $\nu$.

By transposing the operator in (3), we get

$$
p^{1 / 2} J_{\nu}^{2}\left(a x^{1 / 2}\right)=\pi^{-1 / 2} x^{-1 / 2} J_{2 \nu}\left(2 a x^{1 / 2}\right), \quad(\nu>-1 / 2) .
$$

3. Fractional Integration of Expansions in Bessel Functions. The Fourier-Bessel series for $f(x)$ is given by

$$
f(x)=\sum_{n=1}^{\infty} a_{n} J_{\nu}\left(j_{n} t\right) d t, \quad(0<x<1, \nu>-1 / 2),
$$

where

$$
a_{n}=\frac{2}{J_{\nu+1}^{2}\left(j_{n}\right)} \int_{0}^{1} t f(t) J_{\nu}\left(j_{n} t\right) d t
$$

and where $j_{n}$ is the $n$th positive root of $J_{\nu}(x)=0$. From (5) it follows that

$$
x^{-1 / 2} f\left(x^{1 / 2}\right)=\sum_{n=1}^{\infty} a_{n} x^{-1 / 2} J_{\nu}\left(j_{n} x^{1 / 2}\right) .
$$

The conditions on $f(x)$ under which the series (7) is uniformly convergent, or under which at least the sum of $n$ terms of this series remains bounded for all $n$ and all $x$ in the interval $0 \leqq x \leqq 1$, are clearly stated in Lemma 1 and Theorem I of a paper by C. N. Moore.* Assuming that $f(x)$ satisfies these conditions, we can integrate the series (7) fractionally $\dagger$ term by term. We get, applying equation (3),

${ }^{*} \mathrm{C} . \mathrm{N}$. Moore, On the uniform convergence of the developments in Bessel functions, Transactions of this Society, vol. 12 (1911), pp. 181-206.

† W. O. Pennell, loc. cit. 


$$
p^{-1 / 2} x^{-1 / 2} f\left(x^{1 / 2}\right)=\pi^{1 / 2} \sum_{n=1}^{\infty} a_{n} J_{\nu / 2}^{2}\left(\frac{j_{n} x^{1 / 2}}{2}\right) .
$$

If we put

$$
\phi\left(x^{1 / 2}\right)=p^{-1 / 2} x^{-1 / 2} f\left(x^{1 / 2}\right),
$$

then

and

$$
f\left(x^{1 / 2}\right)=x^{1 / 2} p^{1 / 2} \phi\left(x^{1 / 2}\right),
$$

$$
\phi(x)=\pi^{1 / 2} \sum_{n=1}^{\infty} a_{n} J_{\nu / 2}^{2}\left(\frac{j_{n} x}{2}\right), \quad(0<x<1, \nu \geqq 1),
$$

where $a_{n}$ is given by (6).

If we apply the preceding reasoning to the Dini series*

$$
f(x)=\sum_{n=1}^{\infty} b_{n} J_{\nu}\left(\lambda_{n} x\right)
$$

where $\lambda_{n}$ is the $n$th positive root of

$$
z^{-\nu}\left\{z J_{\nu}^{\prime}(z)+H J_{\nu}(z)\right\}=0, \quad(\nu>-1 / 2),
$$

$H$ any constant, and

$$
b_{n}=\frac{2 \lambda_{n}^{2} \int_{0}^{1} t f(t) J_{\nu}\left(\lambda_{n} t\right) d t}{\left(\lambda_{n}^{2}-\nu^{2}\right) J_{\nu}^{2}\left(\lambda_{n}\right)-\lambda_{n}^{2} J_{\nu}^{\prime 2}\left(\lambda_{n}\right)}
$$

we obtain the expansion

$$
\phi(x)=\pi^{1 / 2} \sum_{n=1}^{\infty} b_{n} J_{\nu / 2}^{2}\left(\frac{\lambda_{n} x}{2}\right), \quad(0<x<1, \nu \geqq 1) .
$$

The function $\phi(x)$ is such that if $f\left(x^{1 / 2}\right)=x^{1 / 2} p^{1 / 2} \phi\left(x^{1 / 2}\right)$, then $f(x)$ satisfies the conditions sufficient for a function to be expansible in a Dini series which may be integrated term by term $\dagger$ in the interval $0 \leqq x \leqq 1$

Let $f(x)$ be representable by a Schlömilch series of the type $\ddagger$

$$
f(x)=\pi^{1 / 2} 2^{\nu-1} \sum_{n=1}^{\infty} \frac{a_{n}}{n^{\nu-1}} J_{\nu}(n x),
$$

* See Watson, loc. cit., pp. 597-605.

$\dagger$ C. N. Moore, loc. cit., Lemma 1, Theorem I.

$\ddagger$ Pennell, loc. cit., p. 120. 
which is assumed to be such that it may be integrated term-byterm in the interval $0 \leqq x \leqq \pi$. Making use of the method by which (I) was obtained from (5), we find the expansion

$$
\phi(x)=\pi 2^{\nu-1} \sum_{n=1}^{\infty} \frac{a_{n}}{n^{\nu-1}} J_{\nu / 2}^{2}\left(\frac{n x}{2}\right), \quad(0<x<\pi, \nu \geqq 1),
$$

where

$$
\phi\left(x^{1 / 2}\right)=p^{-1 / 2} x^{-1 / 2} f\left(x^{1 / 2}\right) .
$$

If the above method is applied to Neumann and Kapteyn series, well known expansions in terms of squares of Bessel functions are obtained. Expansions (I), (II), and (III) have seemingly never been published.

The Ohio State University

\section{NOTE CONCERNING GROUP POSTULATES*}

\section{BY RAYMOND GARVER}

Let there be given a set of elements $G(a, b, c, \cdots)$ and a rule of combination, which may be called multiplication, by which any two elements, whether they be the same or different, taken in a specified order, determine a unique result which may or may not be an element of $G$. This system is called a group if it satisfies certain postulates; various sets of postulates have been given by different writers, and such matters as the independence of postulates and relations between sets of postulates have been pretty thoroughly covered. Most of this work was done in this country in the early part of the present century. $\dagger$

It seems, however, that one interesting and rather important

* Presented to the Society, June 20, 1934.

† See Pierpont, Annals of Mathematics, (2), vol. 2 (1900), p. 47; Moore, Transactions of this Society, vol. 3 (1902), pp. 485-492, vol. 5 (1904), p. 549 and vol. 6 (1905), pp. 179-180; Huntington, this Bulletin, vol. 8 (1902), pp. 296-300 and 388-91 and Transactions of this Society, vol. 4 (1903), p. 30, vol. 6 (1905), pp. 34-35 and 181-197; Dickson, Transactions of this Society, vol. 6 (1905), pp. 198-204. 